\title{
From the Mendeleev periodic table to particle physics and back to the periodic table
}

\author{
MAURice R. KibleR \\ Université de Lyon, Institut de Physique Nucléaire, université Lyon 1 and CNRS/IN2P3 \\ 43 Bd du 11 Novembre 1918, F-69622 Villeurbanne Cedex, France
}

\begin{abstract}
We briefly describe in this paper the passage from Mendeleev's chemistry (1869) to atomic physics (in the 1900's), nuclear physics (in 1932) and particle physics (from 1953 to 2006). We show how the consideration of symmetries, largely used in physics since the end of the 1920's, gave rise to a new format of the periodic table in the 1970's. More specifically, this paper is concerned with the application of the group $\mathrm{SO}(4,2) \otimes \mathrm{SU}(2)$ to the periodic table of chemical elements. It is shown how the Madelung rule of the atomic shell model can be used to set up a periodic table that can be further rationalized via the group $\mathrm{SO}(4,2) \otimes \mathrm{SU}(2)$ and some of its subgroups. Qualitative results are obtained from this nonstandard table.
\end{abstract}

PACS: 03.65.Fd, 31.15.Hz

Key words: atomic physics, subatomic physics, group theory, flavor group, periodic table of chemical elements

\section{Introduction}

Antoine Laurent de Lavoisier (1743-1794) is certainly the father of modern chemistry. Nevertheless, the first important progress in the classification of chemical elements is due to Dimitri Ivanovitch Mendeleev (1834-1907). Indeed, the classification of elements did not start with Mendeleev. The impact for chemistry of numerous precursors of Mendeleev is well-known (van Spronsen, 1969; Rouvray and King, 2004; Scerri, 2007). For example we can mention, among others, Johann W. Döbereiner and his triads of elements (1829), Max von Pettenkofer and his groupings of elements (1850), Alexandre E. Beguyer de Chancourtois and his spiral or telluric periodic table (1862), John A.R Newlands and his law of octaves (1864), William Odling and his periodic table (1864), and Julius Lothar Meyer and his curve of atomic volumes (1868). In spite of the interest of the works of his predecessors, Mendeleev is recognized as the originator of the classification of elements for the following reasons. As a matter of fact, in 1869 Mendeleev was successful in four directions: (i) he gave a classification of elements according to growing atomic weights; (ii) he made two inversions ( $\mathrm{Te} / \mathrm{I}$ and $\mathrm{Ni} / \mathrm{Co}$ ) violating the ordering via growing atomic weights and thus compatible with the now accepted ordering via growing atomic numbers; (iii) he predicted the existence of new elements (via the eka-process); and (iv) he described in a qualitative and quantitative way the main chemical and physical properties of the predicted elements. It is true that other scientists tried to develop ideas along the lines (i)-(iv). However, Mendeleev was the first to make observable predictions for new elements. A question naturally arises: What happened after the establishment of the Mendeleev periodic table? There are two answers. 
First, let us mention the development and the extension of the table with the discovery of new elements. For instance, the element called eka-silicon predicted by Mendeleev was discovered in 1886. Such an element, now called germanium, belongs to the same column as silicon (it is located below silicon and above tin in the periodic table with horizontal lines). This discovery illustrates the 'eka'process or 'something is missing'-process: In order to respect some regularity and periodicity arguments, Mendeleev left an empty box at the right of silicon (in his periodic table with vertical lines) and predicted a new element with the correct mass and density.

The discovery of new elements continued during the end of the 19th century, during the whole 20th century and is still the subject of experimental and theoretical investigations. Approximately, 70 elements were known in 1870, 86 in 1940 and 102 in 1958. In the present days, we have 116 elements (the last ones are less and less stable). Some of the recently observed elements do not have a name (the last named is called roentgenium). There is no major reason, except for experimental reasons, to have an end for the periodic table. The research for heavy elements (indeed, superheavy nuclei) is far from being finished.

Second, let us mention a spectacular advance in the understanding of the complexity of matter. This gave rise to the discovery of sub-structures with the advent of classification tables for the constituents of the chemical elements themselves. More precisely, the discovery of atomic structure led to atomic physics at the beginning of the 20th century, then to the birth of nuclear physics in 1932 and, finally, to particle physics in the 1950's. In the present days, subatomic physics deals with the elementary constituents of matter and with the forces or interactions between these constituents.

\section{From chemistry to atomic, nuclear and particle physics}

According to Mendeleev, a chemical element had no internal structure. The chemical elements are made of atoms without constituents. The discovery of the electron by J.J. Thomson in 1897 and that of the atomic nucleus by E. Rutherford in 1911 led to the idea of a planetary model for the atom where the electrons orbit around the nucleus. The simplest nucleus, namely, the proton, was observed by Rutherford in 1919. The simplest atom, the hydrogen atom, is made of a nucleus consisting of a single proton considered as fixed and of an electron moving around the proton. Atomic spectroscopy, seen via the prism of the old theory of quanta (the starting point for quantum mechanics), was born in 1913 when N. Bohr introduced his semi-classical treatment of the hydrogen atom. In 1922, Bohr proposed a building-up principle for the atom based on the planetary Bohr-Sommerfeld model with elliptic orbits and on the filling of each orbit with a maximum of two electrons. This led him to adopt a pyramidal form for the periodic table, that had been already proposed by others like Bayley, and to predict that hafnium is a transition metal as opposed to a rare earth (Scerri, 1994).

A further step towards complexity occurred with the discovery of the neutron 
by J. Chadwick in 1932. A nucleus is made of protons and neutrons, collectively denoted as nucleons. A proton has a positive electric charge, which is the opposite of the electronic charge of the electron, and the neutron has no electric charge. The discovery of a substructure for the nucleus opened the door of nuclear physics. The first model for the description of the strong interactions between nucleons inside the nucleus, the SU(2) model of W. Heisenberg, goes back to 1932. It was soon completed by the prediction by I. Yukawa of the meson $\pi$ in 1933. It can be said that the first version of the strong interaction theory (the ancestor of quantum chromodynamics developed in the 1970's) was born with the works of Heisenberg and Yukawa. In a parallel way, E. Fermi developed in 1933 a theory for the weak interactions inside the nucleus (the ancestor of the electroweak model developed in the 1960's).

In 1932, the situation for emerging particle physics was very simple and symmetrical. At this time, we had four particles: two hadrons (proton and neutron) and two leptons (electron and neutrino), as well as their antiparticles resulting from the P.A.M. Dirac relativistic quantum mechanics introduced in 1928. Remember that the neutrino (in fact the antineutrino of the electron) was postulated by $\mathrm{W}$. Pauli in 1931 in order to ensure the conservation of energy. In some sense, the introduction of the neutrino was made along lines that parallel the eka-process used by Mendeleev. Furthermore, with the discovery of a new lepton, the muon in 1937, of three new hadrons, the pions in 1947-50, and of a cascade of strange hadronic particles (mesons and baryons) in cosmic rays in the 1950's, particle physics was in the 1950's in a situation similar to the one experienced by chemistry in the 1860's. The need for a classification was in order. In this direction, S. Sakata tried without success to introduce in 1956 three elementary particles (proton, neutron, lambda particle) from which it would be possible to generate all hadrons. Indeed, this trial based on the group SU(3) was an extension of the model developed by E. Fermi and C.N. Yang in 1949, based on the group SU(2), with two basic hadrons (proton, neutron).

A decisive step was made with the introduction of the so-called eightfold way by M. Gell-Mann and Y. Ne'eman in 1961. The eightfold way is a model for the classification of hadrons within multiplets (singlets, octets and decuplets) corresponding to some irreducible representation classes (IRCs) of the group SU(3). More precisely, the eight $(0)^{-}$pseudo-scalar mesons and the eight $(1)^{-}$vector mesons were classified in two nonets (nonet $=$ 'octet plus singlet') while the eight $\left(\frac{1}{2}\right)^{+}$baryons were classified in an octet. At this time, we knew nine $\left(\frac{3}{2}\right)^{+}$baryons (the four $\Delta$, the three $\Sigma^{*}$ and the two $\left.\Xi^{*}\right)$. It was not possible to accommodate these nine particles into an 'octet plus singlet'. The closer framework or 'perodic table' for accomodating the nine hadrons was a decuplet with ten boxes. Along the lines of an eka-process, in 1962 Gell-Mann was very well inspired to fill the empty box with a new particle, the particle $\Omega$. He was also able to predict the main characteristics of this postulated particle (spin, parity, isospin, charge, mass, etc.). This particle was observed two years later, in 1964. The interest of group theory for classifying purposes was thus clearly established. The relevance and usefulness of SU(3) was confirmed with the introduction of new particles in 1964: the 'quarks'of M. Gell- 
Mann and the 'aces'of G. Zweig. Gell-Mann and Zweig postulated the existence of three elementary particles and their anti-particles, now called quarks and antiquarks, classified into a triplet and an anti-triplet of $\mathrm{SU}(3)$ from which it is possible to generate all hadrons.

As soon as 1970, a fourth quark was postulated by S.L. Glashow, J. Iliopoulos and L. Maiani to explain the non-observation of certain a priori allowed decays. This quark was indirectly observed in 1974 through the production of a charmed meson so that the matter world was again very symmetrical at that time with four quarks $(u, d, c, s)$ and four leptons $\left(e, \mu, \nu_{e}, \nu_{\mu}\right)$. The interest, for the classification of hadrons, of symmetry groups like $\mathrm{SU}(n)$, now called flavor groups, was then fully confirmed. Besides flavor groups, other groups, called gauge groups, appeared during the 1960's and 1970's to describe interactions between particles. Let us mention the group $\mathrm{SU}(2) \otimes \mathrm{U}(1)$ for the weak and electromagnetic interactions, the group $\mathrm{SU}(3)$ for the strong interactions and the groups $\mathrm{SU}(5), \mathrm{SO}(10)$ and $\mathrm{E}_{6}$ for a grand unified description of electroweak and strong interactions. In addition, the supersymmetric Poincaré group was introduced for unifying external (space-time) symmetries and internal (flavor and gauge) symmetries. As a result of investigations based on symmetries and supersymmetries, we now have in 2006 the standard model and its supersymmetric extensions for describing particles and their interactions (gravitation excluded). This model is based on twelve matter fields, twelve gauge fields mediating interactions between matter fields and one feeding particle (the Higgs boson) which gives mass to massive particles. Indeed, the matter particles (six quarks and six leptons) can be accomotaded in a periodic table with three generations or periods.

The advances in group-theoretical methods in direction of particle physics as well as the introduction of invariance groups and noninvariance groups for describing dynamical systems were a source of inspiration for the use of groups in connection with the periodic table. The rest of this paper is devoted to the building of a periodic table based on the direct product group $\mathrm{SO}(4,2) \otimes \mathrm{SU}(2)$.

\section{Introducing the group $\mathrm{SO}(4,2)$}

Most of the modern presentations of the periodic table of chemical elements are based on a quantum-mechanical treatment of the atom. In this respect, the simplest atom, namely the hydrogen atom, often constitutes a starting point for studying many-electron atoms. Naively, we may expect to construct an atom with atomic number $Z$ by distributing $Z$ electrons on the one-electron energy levels of a hydrogen-like atom. This building-up principle can be rationalized and refined from a group-theoretical point of view. As a matter of fact, we know that the dynamical noninvariance group of a hydrogen-like atom is the special real pseudoorthogonal group in $4+2$ dimensions $\mathrm{SO}(4,2)$ or $\mathrm{SO}(4,2) \otimes \mathrm{SU}(2)$ if we introduce the group SU(2) that labels the spin (Malkin and Man'ko, 1965; Barut and Kleinert, 1967). This result can be derived in several ways. We briefly review two of them (one is well-known, the other is little known). 
The first way corresponds to a symmetry ascent process starting from the geometrical symmetry group $\mathrm{SO}(3)$ of a hydrogen-like atom. Then, we go from $\mathrm{SO}(3)$ to the dynamical invariance group $\mathrm{SO}(4)$ for the discrete spectrum or $\mathrm{SO}(3,1)$ for the continuous spectrum. The relevant quantum numbers for the discrete spectrum are $n, \ell$ and $m_{\ell}$ (with $n=1,2,3, \cdots$; for fixed $n: \ell=0,1, \cdots, n-1$; for fixed $\ell: m_{\ell}=$ $-\ell,-\ell+1, \cdots, \ell)$. The corresponding state vectors $\Psi_{n \ell m_{\ell}}$ can be organized to span multiplets of $\mathrm{SO}(3)$ and $\mathrm{SO}(4)$. The set $\left\{\Psi_{n \ell m_{\ell}}: n\right.$ and $\ell$ fixed $; m_{\ell}$ ranging $\}$ generates an $\mathrm{IRC}$ of $\mathrm{SO}(3)$, noted $(\ell)$, while the set $\left\{\Psi_{n \ell m_{\ell}}: n\right.$ fixed $; \ell$ and $m_{\ell}$ ranging $\}$ generates an IRC of $\mathrm{SO}(4)$. The direct sum

$$
h=\bigoplus_{n=1}^{\infty} \bigoplus_{\ell=0}^{n-1}(\ell)
$$

spanned by all the possible state vectors $\Psi_{n \ell m_{\ell}}$ corresponds to an IRC of the de Sitter group $\mathrm{SO}(4,1)$. The IRC $h$ is also an IRC of $\mathrm{SO}(4,2)$. This IRC thus remains irreducible when restricting $\mathrm{SO}(4,2)$ to $\mathrm{SO}(4,1)$ but splits into two IRC's when restricting $\mathrm{SO}(4,2)$ to $\mathrm{SO}(3,2)$. The groups $\mathrm{SO}(4,2), \mathrm{SO}(4,1)$ and $\mathrm{SO}(3,2)$ are dynamical noninvariance groups in the sense that not all their generators commute with the Hamiltonian of the hydrogen-like atom.

The second way to derive $\mathrm{SO}(4,2)$ corresponds to a symmetry descent process starting from the dynamical noninvariance group $\operatorname{Sp}(8, \mathbf{R})$, the real symplectic group in 8 dimensions, for a four-dimensional isotropic harmonic oscillator. We know that there is a connection between the hydrogen-like atom in $\mathbf{R}^{3}$ and a four-dimensional oscillator in $\mathbf{R}^{4}$ (Kibler and Négadi, 1984). Such a connection can be established via Lie-like methods (local or infinitesimal approach) or algebraic methods based on the so-called Kustaanheimo-Stiefel transformation (global or partial differential equation approach). Both approaches give rise to a constraint and the introduction of this constraint into the Lie algebra of $\operatorname{Sp}(8, \mathbf{R})$ produces a Lie algebra under constraints that turns out to be isomorphic with the Lie algebra of $\mathrm{SO}(4,2)$. From a mathematical point of view, the latter Lie algebra is given by

$$
\operatorname{cent}_{\mathrm{sp}(8, \mathbf{R})} \mathrm{so}(2) / \mathrm{so}(2)=\mathrm{su}(2,2) \sim \mathrm{so}(4,2)
$$

in terms of Lie algebras.

Once we accept that the hydrogen-like atom may serve as a guide for studying the periodic table, the group $\mathrm{SO}(4,2)$ and some of its subgroups play an important role in the construction of this table. This was first realized by Rumer and Fet (Rumer and Fet, 1971) and, independently, by Barut (Barut, 1972). Later, Byakov, Kulakov, Rumer and Fet (Konopel'chenko and Rumer, 1979) further developed this group-theoretical approach of the periodic chart of chemical elements by introducing the direct product $\mathrm{SO}(4,2) \otimes \mathrm{SU}(2)$ and Kibler (Kibler, 2004, 2006) fully described the $\mathrm{SO}(4,2) \otimes \mathrm{SU}(2)$ table in connection with the so-called Madelung rule of atomic spectroscopy. 


\section{The periodic table à la Madelung}

Before introducing the table based on $\mathrm{SO}(4,2) \otimes \mathrm{SU}(2)$, we describe the construction of a periodic table based on the Madelung rule which arises from the atomic shell model. This approach to the periodic table uses the quantum numbers occurring in the quantum-mechanical treatment of the hydrogen atom as well as of a many-electron atom.

Several authors have claimed that the Madelung rule has not been deduced from the first principles of Quantum Mechanics (Löwdin, 1969; Scerri, 2006). This is certainly true if we limit the study of quantum dynamical systems to the paradigmatic quantum systems (and their trivial extensions), namely, the Coulomb and harmonic oscillator systems. However, as shown by Demkov and Ostrosvky (Ostrovsky, 2001) in their remarkable work, it is feasible to deduce the Madelung rule from an effective one-electron potential of a type similar to the one used in the Thomas-Fermi theory of the atom. In some sense, their approach exhibits a phenomenological character. However, the result really follows from $a b$ initio calculations in the framework of nonrelativistic Quantum Mechanics and, from the mathematical point of view, it corresponds to the difficult inverse problem of finding the potential from the spectrum. The approach followed in the present work for presenting (not deriving) the Madelung rule is entirely different. Indeed, we examine the $\mathrm{SO}(3)$ and $\mathrm{SO}(4)$ content of the rule in order to be prepared to pass to the $\mathrm{SO}(4,2)$ and then to the $\mathrm{SO}(4,2) \otimes \mathrm{SU}(2)$ format of the periodic table.

In the central-field approximation, each of the $Z$ electrons of an atom with atomic number $Z$ is partly characterized by the quantum numbers $n, \ell$, and $m_{\ell}$. The numbers $\ell$ and $m_{\ell}$ are the orbital quantum number and the magnetic quantum number, respectively. They are connected to the chain of groups $\mathrm{SO}(3) \supset \mathrm{SO}(2)$ : the quantum number $\ell$ characterizes an IRC, of dimension $2 \ell+1$, of $\mathrm{SO}(3)$ and $m_{\ell}$ a one-dimensional IRC of $\mathrm{SO}(2)$. The principal quantum number $n$ is such that $n-\ell-1$ is the number of nodes of the radial wave function associated with the doublet $(n, \ell)$. In the case of the hydrogen atom or of a hydrogen-like atom, the number $n$ is connected to the group $\mathrm{SO}(4)$ : the quantum number $n$ characterizes an IRC, of dimension $n^{2}$, of $\mathrm{SO}(4)$. The latter IRC splits into the IRC's of $\mathrm{SO}(3)$ corresponding to $\ell=0,1, \cdots, n-1$ when $\mathrm{SO}(4)$ is restricted to $\mathrm{SO}(3)$. A complete characterization of the dynamical state of each electron is provided by the quartet $\left(n, \ell, m_{\ell}, m_{s}\right)$ or alternatively $(n, \ell, j, m)$. Here, the spin $s=\frac{1}{2}$ of the electron has been introduced and $m_{s}$ is the $z$-component of the spin. Furthermore, $j=\frac{1}{2}$ for $\ell=0$ and $j$ can take the values $j=\ell-s$ and $j=\ell+s$ for $\ell \neq 0$. The quantum numbers $j$ and $m$ are connected to the chain of groups $\mathrm{SU}(2) \supset \mathrm{U}(1): j$ characterizes an IRC, of dimension $2 j+1$, of SU(2) and $m$ a one-dimensional IRC of U(1).

Each doublet $(n, \ell)$ defines an atomic shell. The ground state of the atom is obtained by distributing the $Z$ electrons of the atom among the various atomic shells $n \ell, n^{\prime} \ell^{\prime}, n^{\prime \prime} \ell^{\prime \prime}, \cdots$ according to (i) an ordering rule and (ii) the Pauli exclusion principle. A somewhat idealized situation is provided by the Madelung ordering rule: the energy of the shells increases with $n+\ell$ and, for a given value of $n+\ell$, with $n$. This may be depicted by Fig. 1 where the rows are labelled with $n=1,2,3, \cdots$ 
and the columns with $\ell=0,1,2, \cdots$ and where the entry in the $n$-th row and $\ell$-th column is $[n+\ell, n]$. We thus have the ordering $[1,1]<[2,2]<[3,2]<[3,3]<$ $[4,3]<[4,4]<[5,3]<[5,4]<[5,5]<[6,4]<[6,5]<[6,6]<\cdots$. This dictionary order corresponds to the following ordering of the $n \ell$ shells

$$
1 s<2 s<2 p<3 s<3 p<4 s<3 d<4 p<5 s<4 d<5 p<6 s<\cdots,
$$

which is verified to a good extent by experimental data.

\begin{tabular}{|c|c|c|c|c|c|c|}
\hline & 0 & 1 & 2 & 3 & 4 & $\ldots$ \\
\hline 1 & {$[1,1]$} & & & & & \\
\hline 2 & {$[2,2]$} & {$[3,2]$} & & & & \\
\hline 3 & {$[3,3]$} & {$[4,3]$} & {$[5,3]$} & & & \\
\hline 4 & {$[4,4]$} & {$[5,4]$} & {$[6,4]$} & {$[7,4]$} & & \\
\hline 5 & {$[5,5]$} & {$[6,5]$} & {$[7,5]$} & {$[8,5]$} & {$[\ldots]$} & \\
\hline 6 & {$[6,6]$} & {$[\ldots]$} & & & & \\
\hline 7 & {$[\ldots]$} & & & & & \\
\hline$\cdot$ & & & & & & \\
\hline
\end{tabular}

Fig. 1. The $[n+\ell, n]$ Madelung array. The lines are labelled by $n=1,2,3, \cdots$ and the columns by $\ell=0,1,2, \cdots$. For fixed $n$, the label $\ell$ assumes the values $\ell=0,1, \cdots, n-1$.

From these considerations of an entirely atomic character, we can construct a periodic table of chemical elements. We start from the Madelung array of Fig. 1. Here, the significance of the quantum numbers $n$ and $\ell$ is abandoned. The numbers $n$ and $\ell$ are now simple row and column indexes, respectively. We thus forget about the significance of the quartet $n, \ell, j, m$. The various blocks $[n+\ell, n]$ are filled in the dictionary order, starting from $[1,1]$, with chemical elements of increasing atomic numbers. More precisely, the block $[n+\ell, n]$ is filled with $2(2 \ell+1)$ elements, the atomic numbers of which increase from left to right. This yields Fig. 2, where each element is denoted by its atomic number $Z$. For instance, the block $[1,1]$ is filled with $2(2 \times 0+1)=2$ elements corresponding to $Z=1$ up to $Z=2$. In a similar way, the blocks [2,2] and [3,2] are filled with $2(2 \times 0+1)=2$ elements and $2(2 \times 1+1)=6$ elements corresponding to $Z=3$ up to $Z=4$ and to $Z=5$ up to $Z=10$, respectively. It is to be noted, that the so obtained periodic table a priori contains an infinite number of elements: the $n$-th row contains $2 n^{2}$ elements and each column (bounded from top) contains an infinite number of elements. 
M.R. Kibler

\begin{tabular}{|c|c|c|c|c|c|c|}
\hline & $\mathrm{I}=0$ (s-block) & $\mathrm{I}=1$ (p-block) & $\mathrm{I}=2$ (d-block) & $\mathrm{I}=3$ (f-block) & $\mathrm{I}=4$ (g-block) & $\ldots$ \\
\hline$n=1$ & 1 up to 2 & & & & & \\
\hline$n=2$ & 3 up to 4 & 5 up to 10 & & & & \\
\hline$n=3$ & 11 up to 12 & 13 up to 18 & 21 up to 30 & & & \\
\hline$n=4$ & 19 up to 20 & 31 up to 36 & 39 up to 48 & 57 up to 70 & & \\
\hline$n=5$ & 37 up to 38 & 49 up to 54 & 71 up to 80 & 89 up to 102 & 121 up to 138 & \\
\hline$n=6$ & 55 up to 56 & 81 up to 86 & 103 up to 112 & 139 up to 152 & $\ldots$ & \\
\hline$n=7$ & 87 up to 88 & 113 up to 118 & 153 up to 162 & $\ldots$ & & \\
\hline$n=8$ & 119 up to 120 & 163 up to 168 & $\ldots$ & & & \\
\hline$n=9$ & 169 up to 170 & $\ldots$ & & & & \\
\hline$\cdots$ & $\cdots$ & & & & & \\
\hline
\end{tabular}

Fig. 2. The periodic table deduced from the Madelung array. The box $[n+\ell, n]$ is filled with $2(2 \ell+1)$ elements. The filling of the various boxes $[n+\ell, n]$ is done according to the dictionary order implied by Fig. 1.

\section{$5 \quad$ The periodic table $\grave{a}$ la $\mathrm{SO}(4,2) \otimes \mathrm{SU}(2)$}

We are now in a position to give a group-theoretical articulation to the periodic table of Fig. 2. For fixed $n$, the $2(2 \ell+1)$ elements in the block $[n+\ell, n]$, that we shall refer to an $\ell$-block, may be labelled in the following way. For $\ell=0$, the $s$-block in the $n$-th row contains two elements that we can distinguish by the number $m$ with $m$ ranging from $-\frac{1}{2}$ to $\frac{1}{2}$ when going from left to right in the row. For $\ell \neq 0$, the $\ell$-block in the $n$-th row can be divided into two sub-blocks, one corresponding to $j=\ell-\frac{1}{2}$ (on the left) and the other to $j=\ell+\frac{1}{2}$ (on the right). Each sub-block contains $2 j+1$ elements, with $2 j+1=2 \ell$ for $j=\ell-\frac{1}{2}$ and $2 j+1=2(\ell+1)$ for $j=\ell+\frac{1}{2}$, that can be distinguished by the number $m$ with $m$ ranging from $-j$ to $j$ by step of one unit when going from left to right in the row. In other words, a chemical element can be located in the table by the quartet $(n, \ell, j, m)$, where $j=\frac{1}{2}$ for $\ell=0$.

Following Byakov, Kulakov, Rumer and Fet (Konopel'chenko and Rumer, 1979) it is perhaps interesting to use an image with streets, avenues and houses in a city. Let us call Mendeleev city the city whose west-east streets are labelled by $n$ and north-south avenues by $(\ell, j, m)$. In the $n$-th street there are $n$ blocks of houses. The $n$ blocks are labelled by $\ell=0,1, \cdots, n-1$ so that the address of a block is $(n, \ell)$. Each block contains one sub-block (for $\ell=0$ ) or two sub-blocks (for $\ell \neq 0$ ). An address $(n, \ell, j, m)$ can be given to each house: $n$ indicates the street, $\ell$ the 
block, $j$ the sub-block and $m$ the location inside the sub-block. The organization of the city appears in Fig. 3.

At this stage, it is worthwhile to re-give to the quartet $(n, \ell, j, m)$ its grouptheoretical significance. Then, Mendeleev city is clearly associated to the IRC $h \otimes[2]$ of $\mathrm{SO}(4,2) \otimes \mathrm{SU}(2)$ where [2] stands for the fundamental representation of $\mathrm{SU}(2)$. The whole city corresponds to the IRC

$$
\bigoplus_{n=1}^{\infty} \bigoplus_{\ell=0}^{n-1} \bigoplus_{j=\left|\ell-\frac{1}{2}\right|}^{j=\ell+\frac{1}{2}}(j)=\left(\bigoplus_{n=1}^{\infty} \bigoplus_{\ell=0}^{n-1}(\ell)\right) \otimes[2]
$$

of $\mathrm{SO}(4,2) \otimes \mathrm{SU}(2)$ in the sense that all the possible quartets $(n, \ell, j, m)$, or alternatively $\left(n, \ell, m_{\ell}, m_{s}\right)$, can be associated to state vectors spanning this IRC. In the latter equation, $(\ell)$ and $(j)$ stand for the IRC's of $\mathrm{SO}(3)$ and $\mathrm{SU}(2)$ associated with the quantum numbers $\ell$ and $j$, respectively.

We can ask the question: How to move in Mendeleev city? Indeed, there are several bus lines to go from one house to another one? The $\mathrm{SO}(3)$ bus lines, also called $\mathrm{SO}(3) \otimes \mathrm{SU}(2)$ ladder operators, make it possible to go from one house in a given $\ell$-block to another house in the same $\ell$-block. The $\mathrm{SO}(4)$ bus lines, also called $\mathrm{SO}(4) \otimes \mathrm{SU}(2)$ ladder operators, and the $\mathrm{SO}(2,1)$ bus lines, also called $\mathrm{SO}(2,1)$ ladder operators, allow to move in a given street and in a given avenue, respectively. Finally, it should be noted that there are taxis, also called $\mathrm{SO}(4,2) \otimes \mathrm{SU}(2)$ ladder operators, to go from a given house to an arbitrary house.

Another question concerns the inhabitants, also called chemical elements, of Mendeleev city. In fact, they are distinguished by a number $Z$, also called atomic number. The inhabitant living at the address $(n, \ell, j, m)$ has the number

$$
\begin{aligned}
Z(n \ell j m) & =\frac{1}{6}(n+\ell)\left[(n+\ell)^{2}-1\right]+\frac{1}{2}(n+\ell+1)^{2}-\frac{1}{4}\left[1+(-1)^{n+\ell}\right] \\
& \times(n+\ell+1)-4 \ell(\ell+1)+\ell+j(2 \ell+1)+m-1 .
\end{aligned}
$$

Each inhabitant may also have a nickname. All the inhabitants up to $Z=110$ have a nickname. For example, we have Ds, or darmstadtium in full, for $Z=110$. Not all the houses in Mendeleev city are inhabited. The inhabited houses go from $Z=1$ to $Z=116$ (the houses $Z=113$ and $Z=115$ are occupied since the beginning of 2004). The houses corresponding to $Z \geq 117$ are not presently inhabited. When a house is not inhabited, we also say that the corresponding element has not been observed yet. The houses from $Z=111$ to $Z=116$ are inhabited but have not received a nickname yet. The various inhabitants known at the present time are indicated on Fig. 4.

It is not forbidden to get married in Mendeleev city. Each inhabitant may get married with one or several inhabitants (including one or several clones). For example, we know $\mathrm{H}_{2}$ (including $\mathrm{H}$ and its clone), $\mathrm{HCl}$ (including $\mathrm{H}$ and $\mathrm{Cl}$ ), and $\mathrm{H}_{2} \mathrm{O}$ (including $\mathrm{O}, \mathrm{H}$ and its clone). However, there is a strict rule in the city: the assemblages or married inhabitants have to leave the city. They must live in another city and go to a city sometimes referred to as a molecular city. Only the clones may stay in Mendeleev city. 


\section{Qualitative aspects of the $\mathrm{SO}(4,2) \otimes \mathrm{SU}(2)$ periodic table}

Going back to Physics and Chemistry, we now describe Mendeleev city as a periodic table for chemical elements. We have obtained a table with rows and columns for which the $n$-th row contains $2 n^{2}$ elements and the $(\ell, j, m)$-th column contains an infinite number of elements. A given column corresponds to a family of chemical analogs, as in the standard periodic table, and a given row may contain several periods of the standard periodic table.

The chemical elements in their ground state are considered as different states of atomic matter: each atom in the table appears as a particular partner for the (infinite-dimensional) unitary irreducible representation $h \otimes[2]$ of the group $\mathrm{SO}(4,2) \otimes \mathrm{SU}(2)$, where $\mathrm{SO}(4,2)$ is reminiscent of the hydrogen atom and $\mathrm{SU}(2)$ is introduced for a doubling purpose. In fact, it is possible to connect two partners of the representation $h \otimes[2]$ by making use of shift operators of the Lie algebra of $\mathrm{SO}(4,2) \otimes \mathrm{SU}(2)$. In other words, it is possible to pass from one atom to another one by means of raising or lowering operators. The internal dynamics of each element is ignored. In other words, each neutral atom is assumed to be a noncomposite physical system. By way of illustration, we give a brief description of some particular columns and rows of the table.

The alkali-metal atoms are in the first column (with $\ell=0, j=\frac{1}{2}, m=-\frac{1}{2}$, and $n=1,2, \cdots)$; in the atomic shell model, they correspond to an external shell of type $1 s, 2 s, 3 s, \cdots$; we note that hydrogen $(\mathrm{H})$ belongs to the alkali-metal atoms. The second column (with $\ell=0, j=\frac{1}{2}, m=\frac{1}{2}$, and $n=1,2, \cdots$ ) concerns the alkaline earth metals with an external atomic shell of type $1 s^{2}, 2 s^{2}, 3 s^{2}, \cdots$; we note that helium $(\mathrm{He})$ belongs to the alkaline earth metals. The sixth column corresponds to chalcogens (with $\ell=1, j=\frac{3}{2}, m=-\frac{1}{2}$, and $n=2,3, \cdots$ ) and the seventh column to halogens (with $\ell=1, j=\frac{3}{2}, m=\frac{1}{2}$, and $n=2,3, \cdots$ ); it is to be observed that hydrogen does not belong to halogens as it is often the case in usual periodic tables. The eighth column (with $\ell=1, j=\frac{3}{2}, m=\frac{3}{2}$, and $n=2,3, \cdots$ ) gives the noble gases with an external atomic shell of type $2 p^{6}, 3 p^{6}, 4 p^{6}, \cdots ;$ helium, with the atomic configuration $1 s^{2} 2 s^{2}$, does not belong to the noble gases in contrast with usual periodic tables.

The $d$-blocks with $n=3,4$ and 5 yield the three familiar transition series: the iron group goes from $\mathrm{Sc}(21)$ to $\mathrm{Zn}(30)$, the palladium group from $\mathrm{Y}(39)$ to $\mathrm{Cd}(48)$ and the platinum group from $\mathrm{Lu}(71)$ to $\mathrm{Hg}(80)$. A fourth transition series goes from $\operatorname{Lr}(103)$ to $Z=112$ (observed but not named yet). In the shell model, the four transition series correspond to the filling of the $n d$ shell while the $(n+1) s$ shell is fully occupied, with $n=3$ (iron group series), $n=4$ (palladium group series), $n=5$ (platinum group series) and $n=6$ (fourth series). The two familiar inner transition series are the $f$-blocks with $n=4$ and $n=5$ : the lanthanide series goes from $\mathrm{La}(57)$ to $\mathrm{Yb}(70)$ and the actinide series from $\mathrm{Ac}(89)$ to $\mathrm{No}(102)$. Observe that lanthanides start with $\mathrm{La}(57)$ not $\mathrm{Ce}(58)$ and actinides start with $\mathrm{Ac}(89)$ not $\mathrm{Th}(90)$. We note that lanthanides and actinides occupy a natural place in the table and are not reduced to appendages as it is generally the case in usual periodic tables in 18 columns. A superactinide series is predicted to go from $Z=139$ to 
$Z=152$ (and not from $Z=122$ to $Z=153$ as predicted by G.T. Seaborg). In a shell model approach, the inner transition series correspond to the filling of the $n f$ shell while the $(n+2) s$ shell is fully occupied, with $n=4$ (lanthanides), $n=5$ (actinides) and $n=6$ (superactinides). In contrast with Seaborg predictions, the table in Fig. 4 shows that the elements from $Z=121$ to $Z=138$ form a new period having no homologue among the known elements.

In Section 5, we have noted that each $\ell$-block with $\ell \neq 0$ gives rise to two subblocks. As an example, the $f$-block for the lanthanides is composed of a sub-block, corresponding to $j=\frac{5}{2}$, from $\mathrm{La}(57)$ to $\mathrm{Sm}(62)$ and another one, corresponding to $j=\frac{7}{2}$, from $\mathrm{Eu}(63)$ to $\mathrm{Yb}(70)$. This division corresponds to the classification in light or ceric rare earths $\left(j=\frac{5}{2}\right)$ and heavy or yttric rare earths $\left(j=\frac{7}{2}\right)$. It has received justifications both from the experimental (Pascal, 1960) and the theoretical (Oudet, 1979) sides.

From a qualitative point of view, the new aspects to come out of this theoretical analysis can be summed up as follows: (i) hydrogen and helium naturally occur in the first and second columns, respectively; (ii) the inner transition series ( $d$-block), the transition series ( $f$-block) and the $g$-block occupy a natural place in the table (they are not relegated at the periphery of the table); (iii) each of the latter blocks (as well as the $p$-block) exhibits a division into two sub-blocks that is reminiscent of the relativistic splitting $(\ell) \rightarrow\left(\ell-\frac{1}{2}\right) \oplus\left(\ell+\frac{1}{2}\right)$ which should be significant for heavy elements, cf. the distinction between ceric earths and yttric earths (Pascal, 1960); (iv) the number of elements afforded by the table is a priori infinite, in view of the infinite-dimensional irreducible representation of $\mathrm{SO}(4,2)$ on which the table is based (observable elements and/or particles correspond to only a few of the allowed quantum mechanical states).

Another radical outcome from this approach concern the possibility of using group theory from a quantitative point of view. Chemists are very familiar with the use of group-theoretical methods for deriving qualitative results (vibration modes, level splitting, selection rules, etc.). We give in the following section the main lines of a research programme for quantitatively exploiting the potential forces of $\mathrm{SO}(4,2) \otimes \mathrm{SU}(2)$.

\section{Quantitative aspects of the $\mathrm{SO}(4,2) \otimes \mathrm{SU}(2)$ periodic table}

To date, the use of $\mathrm{SO}(4,2)$ or $\mathrm{SO}(4,2) \otimes \mathrm{SU}(2)$ in connection with periodic charts has been limited to qualitative aspects only, viz., classification of neutral atoms and ions as well. We would like to give here the main lines of a programme under development (inherited from nuclear physics and particle physics) for dealing with quantitative aspects.

The first step concerns the mathematics of the programme. The direct product group $\mathrm{SO}(4,2) \otimes \mathrm{SU}(2)$ is a Lie group of order eighteen. Let us first consider the $\mathrm{SO}(4,2)$ part which is a semi-simple Lie group of order $r=15$ and of rank $\ell=3$. It has thus fifteen generators involving three Cartan generators (i.e., generators commuting between themselves). Furthermore, it has three invariant operators or 
M.R. Kibler

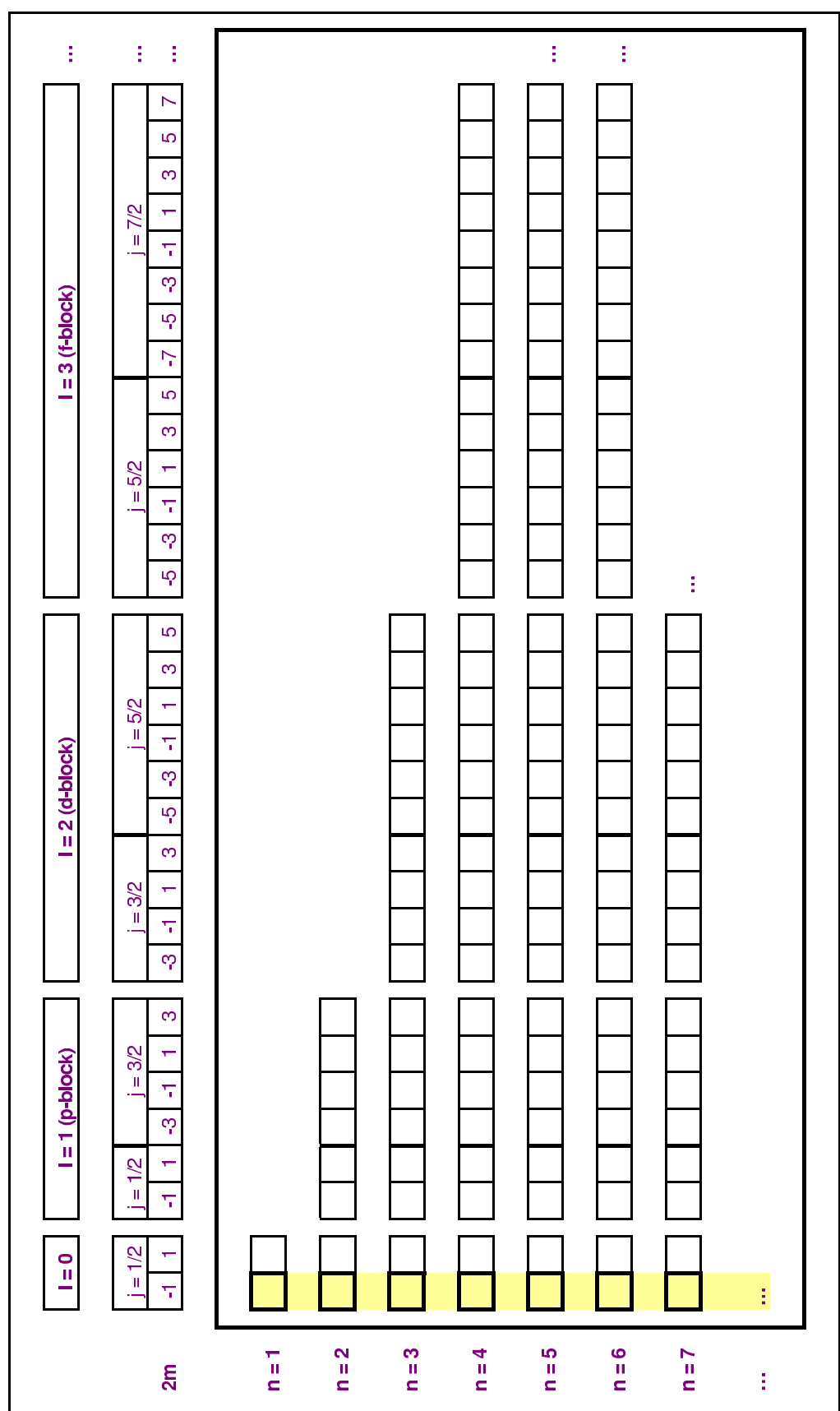

Fig. 3. Mendeleev city. The streets are labelled by $n \in \mathbf{N}^{*}$ and the avenues by $(\ell, j, m)$ $\left[\ell=0,1, \cdots, n-1 ; j=\frac{1}{2}\right.$ for $\ell=0, j=\ell-\frac{1}{2}$ or $j=\ell+\frac{1}{2}$ for $\left.\ell \neq 0 ; m=-j,-j+1, \cdots, j\right]$. 
From the Mendeleev periodic table ...

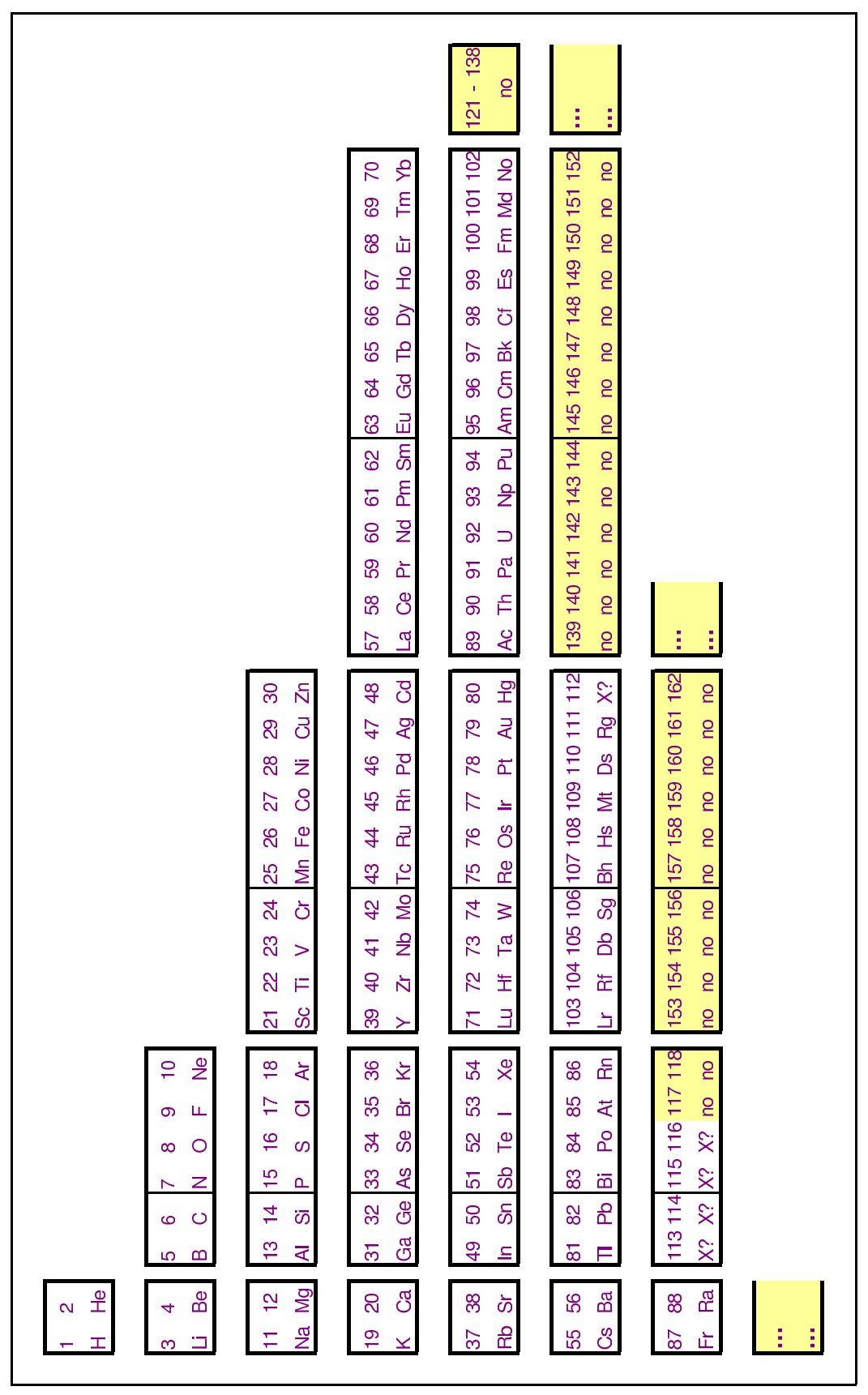

Fig. 4. The inhabitants of Mendeleev city. The houses up to number $Z=116$ are inhabited ['X?' means inhabited (or observed) but not named, 'no' means not inhabited (or not observed)]. 
Casimir operators (i.e., independent polynomials, in the enveloping algebra of the Lie algebra of $\mathrm{SO}(4,2)$, that commute with all generators of the group $\mathrm{SO}(4,2)$ ). Therefore, we have a set of six $(3+3)$ operators that commute between themselves: the three Cartan generators and the three Casimir operators. Indeed, this set is not complete from the mathematical point of view. In other words, the eigenvalues of the six above-mentioned operators are not sufficient for labelling the state vectors in the representation space of $\mathrm{SO}(4,2)$. According to a not very well-known result, popularized by Racah, we need to find $\frac{1}{2}(r-3 \ell)=3$ additional operators in order to complete the set of the six preceding operators. This yields a complete set of nine $(6+3)$ commuting operators and this solves the state labelling problem for the group $\mathrm{SO}(4,2)$. The consideration of the group $\mathrm{SU}(2)$ is trivial: $\mathrm{SU}(2)$ is a semi-simple Lie group of order $r=3$ and of rank $\ell=1$ so that $\frac{1}{2}(r-3 \ell)=0$ in that case. As a result, we end up with a complete set of eleven $(9+2)$ commuting operators.

The second step establishes contact with chemical physics. Each of the eleven operators can be taken to be self-adjoint and thus, from the quantum-mechanical point of view, can describe an observable. Indeed, four of the eleven operators, namely, the three Casimir operators of $\mathrm{SO}(4,2)$ and the Casimir operator of $\mathrm{SU}(2)$, serve for labelling the representation $h \otimes[2]$ of $\mathrm{SO}(4,2) \otimes \mathrm{SU}(2)$ for which the various chemical elements are partners. The seven remaining operators can thus be used for describing chemical and physical properties of the elements, as for instance: ionization energy; oxidation degree; electron affinity; electronegativity; melting and boiling points; specific heat; atomic radius; atomic volume; density; magnetic susceptibility; solubility; etc. In most cases, this can be done by expressing a chemical observable associated with a given property (for which we have few experimental data) in terms of the seven operators which serve as an integrity basis for the various observables. Each observable can be developed as a linear combination of operators constructed from the integrity basis. This is reminiscent of group-theoretical techniques used in nuclear and atomic spectroscopy (cf. the Interacting Boson Model) or in hadronic spectroscopy (cf. the Gell-Mann/Okubo mass formulas for baryons and mesons).

The last step is to proceed with a diagonalization process and then to fit the various linear combinations to experimental data. This can be achieved through fitting procedures concerning either a period of elements, taken along a same line of the periodic table, or a family of elements, taken along a same column of the periodic table. For each property this will lead to a formula or phenomenological law that can be used in turn for making predictions concerning the chemical elements for which no data are available. In addition, it is hoped that this will shed light on regularities and well-known as well as recently discovered patterns of the periodic table, such as unexpected patterns connecting elements via a knight's move in the table (Laing, 2004; Rayner-Canham, 2004). 
From the Mendeleev periodic table...

\section{Closing remarks}

Group-theoretical methods based on symmetry considerations have been continuously developed during the 20th century in order to classify the constituents of matter and to understand their interactions. The $\mathrm{SO}(4,2) \otimes \mathrm{SU}(2)$ periodic table presented in this article was set up along lines similar to the ones used for classifying fundamental particles via flavor groups. The group $\mathrm{SO}(4,2) \otimes \mathrm{SU}(2)$ is a flavor group in the sense that each chemical element appears to be a particular state (or flavor) of a single element.

We close this paper with two remarks. Possible extensions of the work presented in Sections 5-7 concern isotopes and molecules. The consideration of isotopes needs the introduction of the number of nucleons in the atomic nucleus. With such an introduction we have to consider other dimensions for Mendeleev city: the city is no longer restricted to spread in Flatland. Group-theoretical analyses of periodic systems of molecules can be achieved by considering direct products involving several copies of $\mathrm{SO}(4,2) \otimes \mathrm{SU}(2)$. Several works have been already devoted to this subject (Kibler, 2006).

\section{Acknowledgements}

Thanks are due to the Referee for pertinent criticism and useful suggestions.

\section{References}

[1] Barut, A.O. Group Structure of the Periodic System. In B.G. Wybourne, editor, The Structure of Matter. University of Canterbury Publications, Christchurch, New Zealand, pp. 126-136, 1972.

[2] Barut, A.O. and H. Kleinert. Transition Form Factors in the H Atom. Phys. Rev., 160:1149-1151, 1967.

[3] Kibler, M.R. On the Use of the Group $\mathrm{SO}(4,2)$ in Atomic and Molecular Physics. Mol. Phys., 102:1221-1230, 2004.

[4] Kibler, M.R. A group-Theoretical Approach to the Periodic Table: Old and New Developments. In D.H. Rouvray and R.B. King, editors, The Mathematics of the Periodic Table. Nova Science, NY, pp. 237-263, 2006.

[5] Kibler, M. and T. Négadi. Connection between the Hydrogen Atom and the Harmonic Oscillator. Phys. Rev. A, 29:2891-2894, 1984.

[6] Konopel'chenko, V.G. and Yu.B. Rumer. Atoms and Hadrons (Classification Problems). Sov. Phys. Usp., 22:837-840, 1979.

[7] Laing, M. Patterns in the Periodic Table - Old and New. In D.H. Rouvray and R.B. King, editors, The Periodic Table: Into the 21st Century. Research Studies Press, Baldock, U.K., pp. 123-140, 2004.

[8] Löwdin, P.-O. Some Comments on the Periodic System of the Elements. Int. J. Quantum Chem., Quantum Chem. Symp., 3:331-334, 1969.

[9] Malkin, I.A. and V.I. Man'ko. Symmetry of the Hydrogen Atom. Sov. Phys. JETP Lett., 2:146-148, 1965. 
M.R. Kibler: From the Mendeleev periodic table ...

[10] Ostrovsky, V.N. What and How Physics Contributes to Understanding the Periodic Law. Found. Chem., 3:145-182, 2001.

[11] Oudet, X. Valency, Ionicity and Electronic Configuration in Rare Earths. J. Physique (Paris), 40(C5):395-397, 1979.

[12] Pascal, P. Nouveau traité de chimie minérale. Masson, Paris, 1960.

[13] Rayner-Canham, G.W. The Richness of Periodic Patterns. In D.H. Rouvray and R.B. King, editors, The Periodic Table: Into the 21st Century. Research Studies Press, Baldock, U.K., pp. 161-186, 2004.

[14] Rouvray, D.H. and R.B. King. The Periodic Table: Into the 21st Century. Research Studies Press, Baldock, U.K., 2004.

[15] Rumer, Yu.B. and A.I. Fet. The Group Spin(4) and the Mendeleev System. Teoret. Mat. Fiz., 9:203-210, 1971.

[16] Scerri, E. Prediction of the Nature of Hafnium from Chemistry, Bohr's Theory and Quantum Theory. Ann. Sc., 51:137-150, 1994.

[17] Scerri, E. Commentary on Allen \& Knight's Response to the Löwdin Challenge. Found. Chem., 8:285-293, 2006.

[18] Scerri, E. The Periodic Table: Its Story and Its Significance. Oxford University Press, New York, NY, 2007.

[19] van Spronsen, J.W. The Periodic System of Chemical Elements: A History of the First Hundred Years. Elsevier, Amsterdam, 1969. 